\title{
Choice of State Estimation Solution Process for Medium Voltage Distribution Systems
}

\author{
N. Nusrat, M. R. Irving, Senior Member, IEEE and G. A. Taylor, Senior Member, IEEE
}

\begin{abstract}
As distribution networks are turning into active systems, enhanced observability and continuous monitoring becomes essential for effective management and control. The state estimation (SE) tool is therefore now considered as the core component in future distribution management systems. The development of a novel distribution system $\mathrm{SE}$ tool is required to accommodate small to very large networks, operable with limited real time measurements and able to execute the computation of large volumes of data in a limited time frame. In this context, the paper investigates the computation time and voltage estimation qualities of three different SE optimization solution methods in order to evaluate their effectiveness as potential distribution $\mathrm{SE}$ candidate solutions.
\end{abstract}

Index Terms-- Distribution Network, State Estimation, Weighted Least Squares, Weighted Error Modulus.

\section{INTRODUCTION}

The new generation distribution networks are called 'active' as there will be numerous distributed generators (DG), active loads and henceforth bidirectional power flow through the medium voltage (MV) networks. The transition from a passive to an active and smart distribution network is encouraging development to improve the performance and the flexibility of network operation. The active distribution network can improve and maintain quality of service, reduce costs and increase the capacity of the grid to host DG. The complete observation of the states of the system can enable these smart grid functionalities and the state estimation(SE) tool is the core component for this.

SE tools sit in the heart of active distribution network management systems to estimate the actual network status, to feed into control functions, asset management software, demand management tools and some other functionalities. The algorithms and procedures of MV distribution system SE (DSSE) tool require to be capable of enduring heavy computational burden to achieve the required near to real-time state estimation. Compared to the optimization processes applied to transmission system SE may not bring such accuracy for DSSE. Research into DSSE has proposed diverse optimization techniques. A. K. Ghosh et el. applied a

This work is a part of the research project entitled 'High Performance Computing Technologies for Smart Distribution Network Operation (HiPerDNO) that is funded by the European Community's Seventh Framework Programme (FP7/2007-2013).

Nazia Nusrat, Malcolm Irving and Gareth Taylor are with Brunel Institute of Power System, Brunel University, Uxbridge, London, United Kingdom. (email: nazia.nusrat01@brunel.ac.uk, malcolm.irving@brunel.ac.uk, gareth.taylor@brunel.ac.uk ) probabilistic approach for the distribution circuit state estimation based on forward and backward sweeps [1]. R. Hoffman in [2] applied a similar load flow based estimation technique that is referred to as a ladder algorithm. The algorithm converts all measurements into current measurements. The current measurements data are extensively studied by M. E. Baran and A. W. Kelley. These authors adopt a weighted least squares (WLS) approach to develop a $3 \varphi$ DSSE tool [3]. However, the same authors later have developed a branch-current based $3 \varphi$ DSSE tool in order to achieve more computational efficiencies and less sensitivity to line parameters than the conventional node voltage based tools [4]. Reference [5] also applies a $3 \varphi$ current based estimator that uses a current based formulation. A revised version of a branch current based estimation tool is developed by $\mathrm{H}$. Wang and $\mathrm{N}$. S. Schulz using current magnitude and phase angle as the primary states [6]. W. Xu et el. [7] develop a WLS optimization problem where the weight of the measurements is termed as quality tag. F. Bignucolo et el. [8] develop a probabilistic voltage state estimation taking into consideration high penetration of DG. R. Sing et el. [9] investigate compatibility of three different mathematical optimization algorithms (WLS, Weighted Least Absolute Values (WLAV) and Schweppe Huber Generalized M (SHGM) estimators) for DSSE with UK generic distribution networks in presence of DG. Many papers have considered the virtual measurements as an equality constraint that enables reduction of ill conditioning problem to some extent [8] [9] [10] [11] [12].

In this paper, we aim to assess three SE solution process as candidate DSSE solution. The optimization processes include classical and constrained WLS as well as a comparatively novel approach referred to as weighted error modulus (WEM).

\section{Power System State Estimation}

Power system SE is a tool that estimates the actual system state through some mathematical optimization procedures utilizing metered data which are prone to small or large errors. The calculated system state will comply with as much of the available measurement information as possible. The SE is a minimization problem that satisfies (1). Here $z_{i}$ is the measurement of state $i, h_{i}(x)$ is corresponding measurement, $x$ is the state vector and $M$ is the number of available measurements.

$$
\operatorname{Min} \sum_{i=1}^{M}\left[z_{i}-h_{i}(x)\right]
$$


The measurements are classified into three types: pseudo, real and virtual measurements [9][11] [13].

- The real measurements are the sensor data. The trust level depends on the precision of the metering device.

- Virtual measurements are not measured, but confidently known e.g. zero injection nodes; considered as the most accurate measurements.

- The pseudo measurements are assumed or predicted from the load profiles; these are expected to be the most erroneous.

Each measurement is associated with a weighting factor to quantify the degree of trust for that type of measurement. A weighting factor is calculated from the inverse of the corresponding measurement variance.

\section{MAJOR SE RELATED ISSUES FOR MV DISTRIBUTION SYSTEMS}

The MV distribution networks are typically extensive networks $(100,000 \mathrm{~s} \mathrm{~km}$ long) consisting of hundreds of thousands of nodes. Most of the MV region of the networks are poorly monitored. There will be low information about the network status in the present and near future distribution management system (DMS). The degree of trust on available information will be different depending on the type of the data and accuracy of the instruments. It is not feasible with regard to the economical and physical aspects to place meters at each node and along each branch for measurements of voltage and power in distribution networks. At the same time, we can be more confident in the outcome of the DSSE tools as more real measurements are available to provide system information. The DSSE tool will be using mostly pseudo-measurements, along with several virtual-measurements and relatively few real measurements.

The severe limitation of real time measurements would be the main challenge in developing the DSSE tool. As a matter of fact, the power system state estimator is capable of producing acceptable results when there is a high redundancy of real measurements. Whereas for distribution systems, not only the redundancy is very low, also the various data origins such as measurements through field sensors and load estimation techniques lead to a new challenge for DMS. Due to the presence of these different types of measurement data associated with three very different range of weighting factors and various branch sizes, the normal-equation based state estimator is prone to matrix ill-conditioning.

In essence, the DSSE is expected to address new generation DMS issues like the impact of DG penetration, ill conditioning problem resulting from normal equation based optimization, heavy computational burden arising from large distribution networks and the impact of smart grids.

\section{CHOICES OF POTENTIAL SE SOLUTION PROCESS}

Three methods are discussed and compared as potential methods for DSSE in this section. The WLS method is the most common and widely used SE solution process. Constrained WLS (CWLS) is an alternative approach to solve WLS optimization. WEM is a novel iteratively re-weighting approach introduced in [13].

\section{A. Weighted Least Square (WLS)}

The most commonly used state estimator is the normal equation based WLS method, which provides an optimal solution when it is fed with known measurement variances and normally distributed measurement errors. It minimizes the weighted difference between the calculated states and the measured values using the following equation:

$$
\operatorname{Min} J(x)=[z-h(x)]^{T} W[z-h(x)]
$$

Where, $J(x)$ is the minimization criteria, $z$ is the sensor information, $h(x)$ is the measurement equations, $W$ is the weighting factor matrix and $x$ is the state vector. The state variable ' $x$ ' normally represents the voltage magnitude and the phase angle of every node in the network. The optimum point is achieved when the gradient of $J(x)$ becomes zero. The complexity of the problem arises as (2) is a nonlinear minimization problem. Equation (2) is solved by GaussNewton iterative method that utilizes Taylor series expansion of the gradient of $J(x)$ for approximate linearization of the problem. The Gauss-Newton method reaches the solution point by iteratively improving the calculated quantity. An update of the state vector $\Delta x$, is produced at each iteration.

$$
\Delta x=\left(H^{T} W H\right)^{-1} H^{\mathrm{T}} W[z-h(x)] .
$$

Here $H$ is the Jacobian of $h(x)$. The convergent point is achieved when all elements of $\Delta x$ become close to zero i.e. almost no change occurs to the calculated state vector in two consecutive iterations. $H^{\mathrm{T}} W H$ is called the gain matrix [14]. The state estimator can redistribute the measurement errors to keep all estimation errors (even for higher erroneous measurements) within acceptable levels. It gives a compromise solution between the more accurate and less accurate measurements satisfying (4). Here the real and the estimated value of states are $\mu$ and $x_{\text {est }}$ respectively,

$$
\sum_{i=1}^{M}\left|z_{i}-h_{i}\left(x_{e s t}\right)\right|=\sum_{i=1}^{M}\left|z_{i}-\mu_{i}\right|
$$

and $\operatorname{Max}\left|z-h\left(x_{e s t}\right)\right|_{i}<\operatorname{Max}|z-\mu|_{i}$, where $i=1 . . M$.

\section{B. Constrained Weighted Least Square (CWLS)}

The CWLS method takes virtual measurement as constraints and hence improves the Gain matrix ill-conditioning problem. The CWLS method can be expressed as below [15] [16] [17] [18], when $r=z-h(x)$ and virtual measurement equation $c(x)$ is expressed as equality constraint.

$$
\begin{aligned}
\text { Min } & J(x)=\frac{1}{2}[z-h(x)]^{T} W[z-h(x)] \\
\text { s.t. } & c(x)=0
\end{aligned}
$$

Solving using a Lagrange multiplier, the following is obtained

$$
\mathcal{L}(x, \lambda)=\frac{1}{2}(z-h(x))^{T} W(z-h(x))-\lambda^{\prime} c(x)
$$


Defining,

$$
\frac{\partial h(x)}{\partial x}=H \quad \text { and } \quad \frac{\partial c(x)}{\partial x}=C
$$

$C$ is the Jacobian of $c(x)$. After deriving 1st order optimality conditions, the linearized Gauss-Newton update equation is obtained as

$$
\left|\begin{array}{cc}
H^{T} W H & C^{T} \\
C & 0
\end{array}\right|\left|\begin{array}{c}
\Delta x \\
-\lambda
\end{array}\right|=\left|\begin{array}{c}
H^{T} W r \\
\Delta c
\end{array}\right|
$$

Here, $\Delta x=\Delta x^{k+1}-\Delta x^{k}$ when $k$ is the present iteration number.

The gain matrix $\left(H^{T} W H\right)$ in (7) excludes virtual measurements and therefore can avoid very high weighting values. This reduces the condition number of the gain matrix considerably.

\section{Weighted Error Modulus (WEM)}

A novel estimator, WEM method is proposed as a candidate DSSE tool. In this approach, the weighting value associated with the measurement is modified iteratively within the WLS method. In addition to the measurement errors, linearization errors from the Taylor series approximation of the optimization equation for $J(x)$ are also present. The Gauss-Newton solution of WLS optimization function with linearization errors le can be written:

$$
H \Delta x=r+l e
$$

Here, $r=z-h(x)=$ vector of residuals. According to the Gauss-Newton principle, the linearization error is negligible provided that the initial guess of the state is close to the actual value. Normally, the measurement error approximates around $1 \%$ of the actual state value and therefore the WLS method gives good estimation under normal conditions. This implies that the residual vector always have a considerably smaller value. However, this will not hold true if any gross error exists in the measurements. In that case, the assumption $H \Delta x \approx r$, is no longer true and therefore, $l e$ in (8) will have a larger value. The proposed WEM method utilizes the characteristics of the variation in le depending on the accuracy of the measurement to reweight the weighting vector. Essentially, the WEM method attempts to reduce general measurement errors by the WLS method and gross errors by the WLAV method.

If $\mathrm{w}_{\mathrm{i}}$ is modified iteratively such that for $\mathrm{k}^{\text {th }}$ iteration

$$
w_{i}^{k+1}=\frac{u_{i}}{\left|(H \Delta x)_{i}-r_{i}\right|^{k}}
$$

Here $u_{i}$ is the measurement re-weighting factor. When $(H \Delta x)_{\mathrm{i}}$ is negligible and $\left|r_{i}\right|^{\mathrm{k}}=\left|r_{i}\right|^{\mathrm{k}+1}$, we can say that

$$
\left|(H \Delta x)_{\mathrm{i}}-r_{i}\right|^{k} \approx\left|(H \Delta x)_{\mathrm{i}}-r_{i}\right|^{k+1} \approx r_{i}^{k+1}
$$

By replacing the value for $w_{i}^{k+1}$ and putting in the minimization problem [19]

$$
\operatorname{Min} \sum_{i=1}^{M} u_{i}\left|r_{i}\right|
$$

As the method consisted of one inner and one outer loop operation, the inner loop iteration is capped at a smaller number of iterations (which is five here) and allowed to terminate even if convergence is not achieved. The outer loop is the main control to decide when the algorithm is satisfying the convergence criteria and should terminate.

\section{TEST}

The DSSE candidate solution processes are applied on the 77 node radial network of the United Kingdom generic distribution system (UKGDS) dataset, which represents UK model distribution networks and is developed as a set of benchmarks for research purposes [20]. The 77 node radial network consists of 76 branches shown in Fig. 1. It is assumed that all real and pseudo-measurement errors are within expected thresholds (real within 1\%, pseudo within 50\% error margin) and the topology parameters value are not widely diverse. In this case, the topology parameters from original data set and measurement calculation from load flow values are considered to represent the true values. Real measurements (1 voltage magnitude and 1 power flow measurements) are assumed to be available at grid supply point, i.e. node 1 in Fig.1.

Although the required number of iterations for various DSSE tools are the same (i.e. three in this case), because of different computation methodologies, the estimation execution time is different in Fig. 2. WLS requires the least computation steps, therefore the computation time it takes is expected to be the minimum for the same number of iterations than that for others. On the other hand, WEM consists of one inner and one outer loop which means the total iterations require are equal to the number of inner loop iteration times the number of out loop iterations. Certainly, WEM is usually expected to take a longer time for SE calculation. CWLS method need more calculation and data processing compared to classical WLS, and this may have some effect on the computation time.

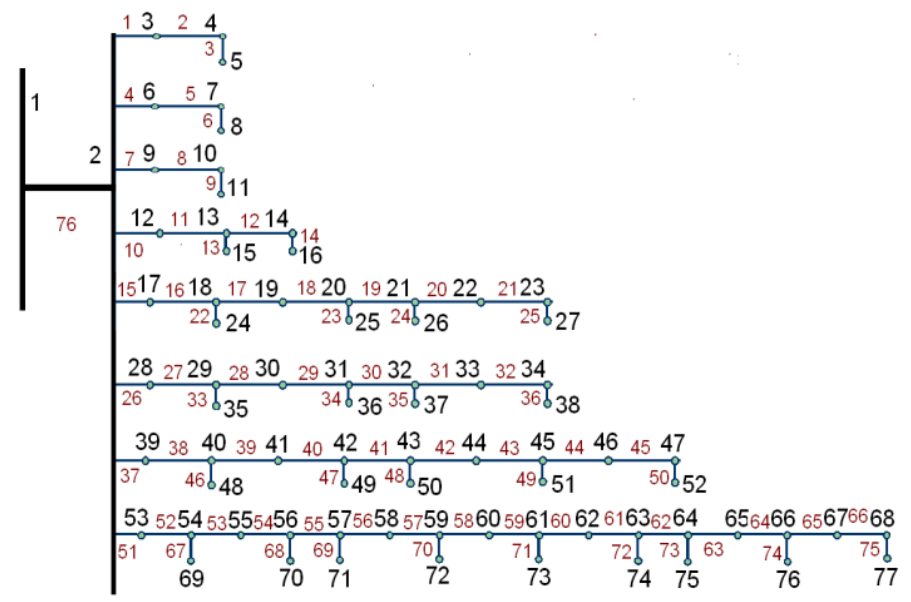

Fig: 77 Node UKGDS network. Bus numbers in black, branch numbers in red. 


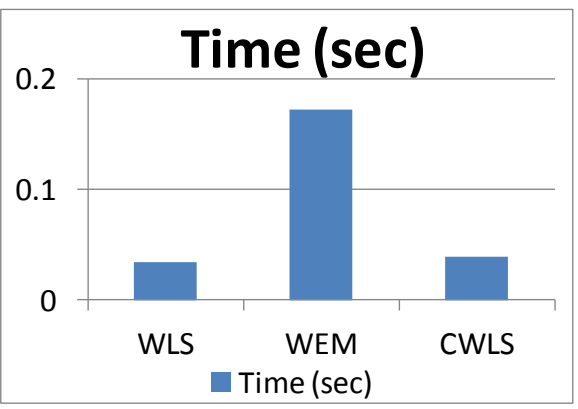

Fig 2: Computation time for three SE solution processes

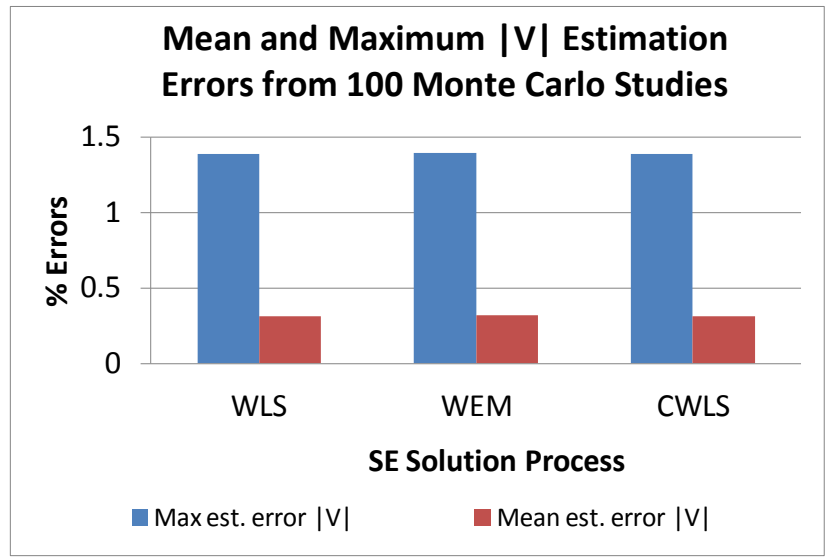

Fig 3: Voltage estimation errors from 100 Mote Carlo studies for three SE solution processes

Voltage estimation errors with respect to load flow results for the state of the network have been plotted for 100 sets of simulated measurement applied to three processes. It is clearly visible that all three processes give the similar quality of estimation for this set of measurements. The mean voltage estimation errors are less than $0.5 \%$ in all cases however maximum voltage estimation errors are around $1.5 \%$.

\section{COMPERATIVE ANALYSIS}

Classical WLS is a popular and widely used optimizer in power system SE problems especially for transmission systems due to its excellent performance in removing errors generated from noisy measurements. However, the application of WLS is more challenging at distribution levels where significant numbers of pseudo and virtual measurements may cause deterioration of the gain matrix condition number. One of the major sources of matrix ill-conditioning is the high weighting factors assigned to virtual measurements. The $W$ matrix in CWLS method does not contain larger values as virtual measurements are used as constraints in the case. They form constrained equations defined by the $C(x)$ matrix which is not included in the normal equations. The $C(x)$ is not squared in solution equation for CWLS. Hence use of virtual measurement as constraints plays an important role to prevent deteriorating condition of the coefficient matrix. However any possibility of the presence of bad data in the enforced constraint may leave negative effects on convergence and estimation quality [14], which is generally an unlikely event to occur. While avoiding forming complete normal equations, the coefficient matrix of CWLS remain no longer positive definite, therefore they require more sophisticated ordering and factorization. This can be treated as a trivial problem to consider as the advanced software technology like MATLAB can resolve this except for the additional computation time that may be required [15] [18].

Although the WLS method can be used to successfully detect and removes outliers, it is not always efficient in detecting and overcoming the effect of gross measurement errors or bad data. WEM method is therefore proposed as a candidate DSSE solution. WEM combines the theory of WLS and WLAV, therefore it can effectively remove both gross errors and small noises from measurement data. WEM tends to adjust the weight with magnitudes of relevant measurement residual values to attribute more emphasis to more coherent measurement data. The method therefore, instead of treating all pseudo-measurements equally, prefers a few of them to gain more weight as the solution approaches convergence. The reweighting factors are expected to promote those pseudomeasurements which are closer representation of real states. However there is a risk of bad estimation if WEM trusts an erroneous pseudo-measurement applying too much weight to it.

The evaluation on the 77 node network shows that all three processes take a similar number of iterations; however computation time for WEM method is significantly higher. In terms of quality of voltage estimation, all three solution processes perform equally well.

\section{CONCLUSION}

In this paper, we have assessed three SE solution processes: classical WLS, CWLS, and WEM methods, as candidate DSSE tools for MV systems. The voltage estimation quality is similar for the 77 node network, however the computation time is longer for WEM solution process. Considering the properties of the three methods, CWLS and WEM have greater potential as candidate DSSE solution processes. Considering the test case results, CWLS can be chosen as the best of the three solution processes to be applied for MV distribution systems.

\section{REFERENCES}

[1] A. Ghosh, D. Lubkeman, M. Downey and R. Jones, "Distribution Circuit State Estimation Using A Probabilistic Approach," IEEE Transactions on Power Systems, vol. 12, no. 1, pp. 45-51, Feb 1997.

[2] R. Hoffman, "Practical State Estimation for Electric Distribution Networks," in Power Systems Conference and Exposition, 2006. PSCE '06. 2006 IEEE PES, Oct-Nov 2006.

[3] M. Baran and A. Kelley, "State Estimation for Real-Time Monitoring of Distribution Systems," IEEE Transactions on Power Systems, vol. 3, no. 9, pp. 1601-1609, Aug 1994.

[4] M. Baran and A. Kelley, "A Branch-Current-Based State Estimation Method for Distribution Systems," IEEE Transactions on Power Systems, vol. 10, no. 1, pp. 483-491, Feb 1995.

[5] C. Lu, J. Teng and W. Liu, "Distribution System State Estimation," IEEE Transactions on Power Systems, vol. 10, no. 1, pp. 229-240, Feb 1995.

[6] H. Wang and N. Schulz, "A Revised Branch Current-Based Distribution System State Estimation Algorithm and Meter Placement Impact," IEEE Transactions on Power Systems, vol. 19, no. 1, pp. 207 - 213, Feb 2004.

[7] W. Xu, D. Liu, R. Lu, J. Qiu, Pan, H. and Z. Wei, "A Novel State Estimation Method Based on Quality Tag for Distribution Networks," in CIRED 20th International Conference and Exhibition on Electricity Distribution - Part 1, Jun 2009.

[8] F. Bignucolo, R. Caldon and M. Valente, "Probabilistic Voltage Estimation for Active Control of Distribution Networks," in CIRED 
19th Internation Conference on Electricity Distribution, May 2007.

[9] R. Sing, B. Pal and R. Jabr, "Choice of Estimator for Distribution System State Estimation," IET Generation, Transmisison and Distribution, vol. 7, no. 3, pp. 666- 678, Jul 2009.

[10] V. Thornley, N. Jenkins, White and S., "State Estimation Applied to Active Distribution Networks with Minimal Measurement," in 15th Power System Computation Conference, 2007.

[11] O. Chillard, S. Grenard, O. Devaux and L. Garcia, "Distribution State Estimation Based on Voltage State Variables: Assessment of Results and Limitations," in CIRED 20th International Conference on Electricity Distribution, Jun 2009.

[12] M. Biserica, Y. Besanger, R. Caire, O. Chilard and P. Deschamps, "Neural Networks to Improve Distribution State Estimation-Volt Var Control Performances," IEEE Transactions on Smart Grid, vol. 3, no. 3 , pp. 1137-1144, Sept 2012.

[13] G. Taylor, M. Irving, N. Nusrat, R. Liao and S. Panchandaram, "Developing Novel Information And Communications Technology Based Solutions For Smart Distribution Network Operation," 2010.

[14] J. Chiou, "A Variable Scaling Hybrid Differential Evolution for Solving Large-Scale Power Dispatch Problems," IET Generation, Transmission \& Distribution, vol. 3, no. 2, pp. 154-163, Feb 2009.

[15] A. Abur and A. Exposito, Power System State Estimation Theory and Implementation, NewYork: Marcel Dekker Inc, 2004, p. 101.

[16] L. Holten, A. Gjelsvik, S. Aam, F. Wu and W. Liu, "Comparison of Different Methods for State Estimation," IEEE Transactions on Power Systems, vol. 3, no. 4, pp. 1798-1804, Nov 1988.

[17] A. Monticelli, "Electric Power System State Estimation," Proceedings of the IEEE, vol. 88, no. 2, pp. 262 - 282, Feb 2000.

[18] A. Monticelli, State Estimation in Electric Power Systems A Generalized Approach, USA: Kluwer Academic Publisher, 1999.

[19] G. Taylor, M. Irving, N. Nusrat, R. Liao and S. Panchandaram, "Smart Distribution Network Operation: Emerging Techniques and Standards," in IEEE Power and Energy Society General Meeting, Jul 2011.

[20] "United Kingdom Generic Distribution System," [Online]. Available: http://monaco.eee.strath.ac.uk/ukgds/. [Accessed Sept. 2011].

\section{BIOGRAPHIES}

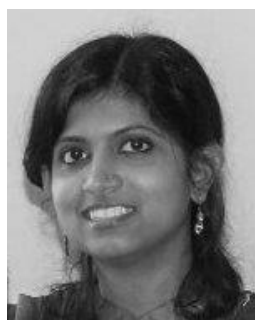

Nazia Nusrat graduated in 2006 with a BSc. degree in Electrical and Electronic Engineering from Bangladesh University of Engineering and Technology (BUET), Bangladesh. She received an MSc in 2009 from Brunel University, UK in Sustainable Electrical Power Engineering. She worked as a research assistant for HiPerDNO project and is currently pursuing a $\mathrm{PhD}$ with Brunel University. Her research interest includes distribution network instrumentation and distribution state estimation.

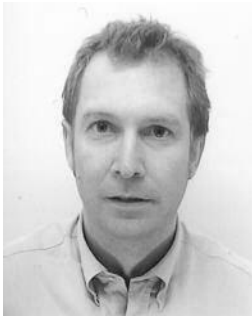

Gareth Taylor (SM' 12) received his BSc degree from the University of London, UK in 1987 and $\mathrm{MSc}$ and $\mathrm{PhD}$ from the University of Greenwich, UK in 1992 and 1997, respectively. He was the National Grid UK post-doctoral scholar at Brunel University, UK from 2000-2003. He is currently a professor of power systems and director of the Brunel Institute of Power Systems, Brunel University, UK. His research interests include smart grids, active network management, power system operation and network optimization.

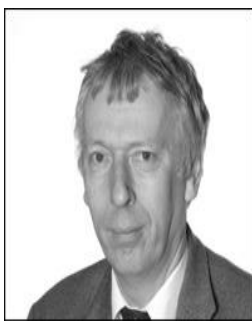

Malcolm Irving (SM' 93) is Professor of Power Systems in the School of Engineering and Design at Brunel University, Uxbridge, UK. He is also Codirector of the Brunel Institute of Power Systems, a major research group in the University specialising in algorithms and software for generation, transmission and distribution systems. He is the author or co-author of more than 150 research publications in the fields of power network analysis and optimal power system operation. 